\title{
ADESIVOS À BASE de TANINOS DAS CASCAS DE DUAS ESPÉCIES DE EUCALIPTO PARA PRODUÇÃO DE CHAPAS DE FLOCOS ${ }^{1}$
}

\author{
Benedito Rocha Vital², Angélica de Cássia Oliveira Carneiro ${ }^{3}$, Alexandre Santos Pimenta ${ }^{2}$, Ricardo Marius
} Della Lucia ${ }^{2}$

\begin{abstract}
RESUMO - Os taninos foram extraídos das cascas de Eucalyptus grandis W. Hill ex Maiden e Eucalyptus pellita F. Muell. com água quente e a adição de $4,5 \%$ de sulfito de sódio, durante três horas, nas temperaturas de 70 e $100^{\circ} \mathrm{C}$, respectivamente. Para a produção dos adesivos, os taninos reagiram com ácido acético e sulfito de sódio, para a redução da viscosidade. Técnicas de DSC (calorimetria diferencial exploratória) foram utilizadas para determinar os parâmetros cinéticos dos adesivos. Foram produzidas, em laboratório, chapas de flocos de Eucalyptus grandis e Pinus elliottii, utilizando-se 8 e $10 \%$ de adesivo de tanino sulfitado e $8 \%$ de adesivo comercial de uréia-formaldeído. As propriedades das chapas foram determinadas segundo a norma ASTM D1037 de 1993. As propriedades das chapas fabricadas apenas com adesivo à base de taninos foram superiores ao mínimo exigido pela norma comercial ANSI/A 208.1-93, com exceção daquelas relacionadas com umidade. As chapas produzidas com adesivos de taninos da casca de Eucalyptus grandis foram superiores àquelas fabricadas com adesivos de taninos da casca de Eucalyptus pellita .
\end{abstract}

Palavras-chave: Taninos condensados, DSC, sulfitação, adesivos.

\section{TWO EUCALYPTS BARK TANNIN-BASED ADHESIVE FOR PRODUCTION OF FLAKEBOARDS}

\begin{abstract}
Eucalyptus grandis and Eucalyptus pellita bark tannins were extracted with hot water, with addition of $4,5 \%$ of sodium sulfite, for a three hours period, at temperatures of $70^{\circ} \mathrm{C}$ and $100^{\circ} \mathrm{C}$, respectively. The tannins were reacted with acetic acid and sodium sulfite, for reduction of adhesive viscosity. Calorimetric exploratory differential techniques were used to determine adhesive kinetic parameters. Flakeboards were fabricated with Pinus elliottii wood flakes, using $8 \%$ or $10 \%$ of Eucalyptus grandis or Eucalyptus pellita tanninbased adhesives or $8 \%$ of commercial urea-formaldehyde adhesive. Board properties were determined according to ASTM D-1037 standard. Except for moisture related properties, the boards fabricated only with tanninbased adhesive were superior to the minimally acceptable ANSI/A 208.1-93 standard. The flakeboards fabricated with Eucalyptus grandis bark tannins were better than those fabricated with Eucalyptus pellita bark tannins.
\end{abstract}

Key words: Condensed tannins, DSC, sulfitation, adhesives

\section{INTRODUÇÃO}

Taninos são substâncias que têm um uso extensivo e longamente estabelecido, mas a definição exata desses constituintes vegetais não é simples. Segundo Waterman e Mole (1994), provavelmente a defini- ção mais aceitável seja a de Bate-Smith e Swain (1962), que classificaram os taninos vegetais como "compostos fenólicos solúveis em água, tendo peso molecular entre 500 e 3.000 e que, ao lado de reações fenólicas usuais, têm a propriedade de precipitar alcalóides, gelatinas e outras proteínas".

\footnotetext{
${ }^{1}$ Recebido para publicação em 01.7.2003 e aceito para publicação em 10.8.2004.

${ }^{2}$ Departamento de Engenharia Florestal da UFV. E.mail: < bvital@ufv.br>.

${ }^{3}$ Programa de Pós-Graduação em Ciência Florestal da UFV. E.mail: <o.carneiro@bol.com.br>.
} 
Os taninos são classificados em dois grupos de compostos químicos de natureza fenólica, os taninos hidrolisáveis e os taninos condensados. O primeiro grupo é encontrado em extratos de cascas e madeiras das árvores de Terminalia, Phyllantus e Caesalpina, dentre outros gêneros, os quais são constituídos de misturas de fenóis simples, como pirogalol e ácido elágico, e também de ésteres do ácido gálico ou digálico com açúcares, principalmente glucose (HERGERT, 1989). Os taninos condensados são compostos cujas unidades fundamentais são estruturas monoméricas de 2fenilbenzopiranos com uma estrutura básica de forma $\mathrm{C}_{6}-\mathrm{C}_{3}-\mathrm{C}_{6}$. A Figura 1 ilustra uma estrutura básica desses taninos.

Os taninos condensados e os flavonóides que lhes dão origem são conhecidos por sua larga distribuição, estando presentes na casca de todas as folhosas e coníferas examinadas até hoje (HERGERT, 1989). Estão presentes ainda, freqüentemente, no cerne de várias essências florestais (HASLAM, 1966; PORTER, 1988). As espécies florestais mais utilizadas para produção comercial de taninos são a acácia-negra (Acacia mearnsii De Wild.) e o quebracho (Schinopsis sp.). No gênero Eucalyptus podem ser citadas as espécies $E$. astringens Maiden, E. wandoo Blakely (HASLAM, 1966) e $E$. grandis (MORI, 2000), entre outras. Esses taninos constituem mais de $90 \%$ da produção mundial de taninos

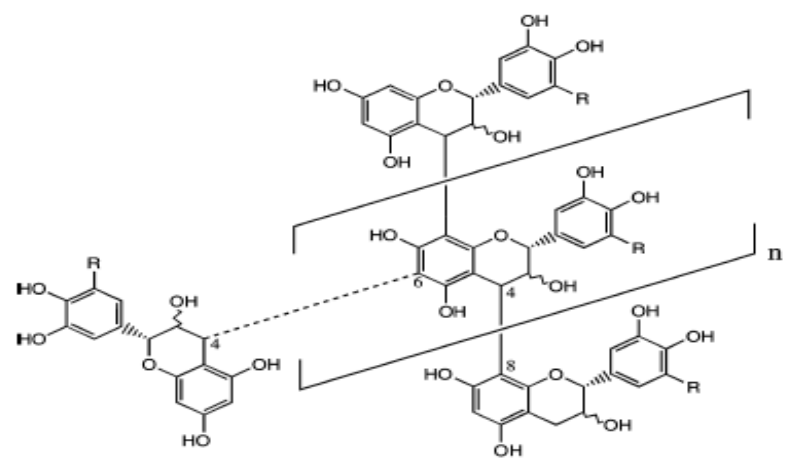

Figura 1 - Modelo de estrutura dos taninos condensados. $\mathrm{Se} \mathrm{R}=\mathrm{H}$ ou $\mathrm{OH}$, essa estrutura representa uma procianilina ou prodelfinidina. A ligação 4-6 (linha pontilhada) é uma alternativa de ligação interflavonóide.

Figure 1 -Structure model for condensed tannins. If $R=H$ or $\mathrm{OH}$, this structure represents a procyanidin or prodelphynidin. Doted 4-6 line is a inter-flavonol bonding.

R. Árvore, Viçosa-MG, v.28, n.4, p.571-582, 2004 comerciais (HERGERT, 1989), sendo adequados, dos pontos de vista químico e econômico, entre outros usos, para a produção de adesivos fenólicos (PIZZI e MITTAL, 1994). Os taninos são extrativos utilizados desde a antigüidade para curtir couro e recentemente vêm sendo empregados na fabricação de adesivos para madeira. Em alguns países, como a Austrália e África do Sul, já são usados há algum tempo em escala comercial (PIZZI, 1983).

Os adesivos à base de taninos-formaldeído de Eucalyptus spp, apresentam, contudo, algumas limitações, como alta viscosidade e baixa resistência da linha de cola. Isso é devido ao fato de os extratos de taninos conterem, além de substâncias fenólicas ativas, outras substâncias, como traços de amino e iminoácidos e, principalmente, açúcares e gomas de alto peso molecular. Outra limitação sua é o tamanho das moléculas tânicas - que são relativamente grandes-,as quais, por isso, têm certa imobilidade (PIZZI, 1994).

Há no Brasil, no entanto, uma grande disponibilidade de cascas dessas espécies, uma vez que são removidas antes de qualquer processamento industrial.

O presente trabalho teve como objetivo geral avaliar o efeito da sulfitação dos taninos das cascas de duas espécies de Eucalyptus nas formulações de adesivas para fabricação de chapas de flocos.

\section{MATERIAL E MÉTODOS}

Os taninos foram extraídos de cascas deEucalyptus grandis provenientes dos plantios da Universidade Federal de Viçosa, em Viçosa, MG, com idade de 20 anos, e das cascas de Eucalyptus pellita provenientes de Bom Despacho, MG (CAF), com idade de 17 anos.

Para determinação do rendimento gravimétrico em taninos, a extração foi feita em autoclave nas temperaturas de $70 \mathrm{e} 100^{\circ} \mathrm{C}$, por um período de três horas, empregandose os seguintes tratamentos: 1) água pura, 2) água $+1,5 \%$ de sulfito de sódio, 3 ) água $+3 \%$ de sulfito de sódio e 4) água $+4,5 \%$ sulfito de sódio (massa/ volume). Foi usada uma relação licor/casca de 20:1 em Eucalyptus grandis e 30:1 em Eucalyptus pellita. Utilizou-se uma relação licor/casca maior para extração dos taninos da casca de E. pellita, porque estes ocupavam maior volume numa mesma massa seca. $\mathrm{O}$ 
Índice de Stiasny dos extratos tânicos foi determinado empregando-se o método utilizado por Mori (1997).

Após a quantificação dos rendimentos gravimétricos foi realizada a extração de taninos da casca de Eucalyptus grandis e Eucalyptus pellita, em quantidade suficiente para a elaboração de adesivos.

A sulfitação foi realizada em um balão de reação, onde foram colocados, sob agitação mecânica constante e aquecimento, $50 \mathrm{~g}$ de taninos (peso de matéria seca), 50 g de água destilada, 2,5 mL de ácido acético e 5\% de sulfito de sódio. Essa mistura foi mantida sob refluxo durante 90 minutos. Para sulfitação dos taninos de E. pellita, utilizaram-se $75 \mathrm{~g}$ de água, uma vez que a viscosidade dos adesivos dessa espécie foi mais elevada, impossibilitando a utilização na pistola pneumática.

Para produzir os adesivos, foram adicionados aos taninos sulfitados $10 \%$ de formaldeído, sendo determinado o teor de sólidos, $\mathrm{pH}$, tempo de gelatinização e viscosidade. O tempo de gelatinização foi obtido de acordo com Mori (2000) e a viscosidade, através de um viscosímetro Thomas Stormer. A quantidade de adesivo foi de aproximadamente $100 \mathrm{~mL}$, com três repetições.

As medidas termoanalíticas foram obtidas com um aparelho DSC 50 da Shimadzu e analisadas por um programa fornecido no equipamento, contendo o modelo cinético de Ozawa. As amostras foram acondicionadas em cápsulas de aço inox capazes de resistir a pressões de vapor da ordem de 25 bar. Calibrações de temperatura e entalpia foram estabelecidas com o elemento químico Índio. Foram analisados $8 \mathrm{mg}$ de amostra líquida, sendo os termogramas obtidos da temperatura ambiente até $250^{\circ} \mathrm{C}$, com taxas de aquecimento de 5, 10 e $15^{\circ} \mathrm{C} /$ minuto. Após conseguir os dados nos três incrementos de temperatura, procedeu-se às análises das curvas, para obtenção dos parâmetros cinéticos.

Chapas de flocos foram fabricadas com 8 e $10 \%$ de adesivos tânicos. Foram fabricadas, também, chapas com $8 \%$ do adesivo comercial à base de uréia-formadeído (Cascamite PB 2346 da ALBA-Química), que serviram como base para comparação. Os teores de adesivo foram calculados com base no seu teor de sólidos em relação à massa anidra de madeira.
As dimensões finais das chapas foram iguais a $40 \mathrm{~cm}$ X $40 \mathrm{~cm} \mathrm{X} \mathrm{1,00} \mathrm{cm.} \mathrm{A} \mathrm{densidade} \mathrm{final} \mathrm{desejada}$ foi de $0,56 \mathrm{~g} / \mathrm{cm}^{3}$ para Pinus elliottii e de $0,70 \mathrm{~g} / \mathrm{cm}^{3}$ para Eucalyptus grandis, mantendo-se uma razão de compactação igual a 1,4. Empregou-se uma pressão de $32 \mathrm{kgf} / \mathrm{cm}^{2}$, temperatura de prensagem de $170{ }^{\circ} \mathrm{C}$ e tempo de oito minutos e 30 segundos.

No Quadro 1, mostram-se os tratamentos empregados.

As propriedades mecânicas e físicas foram determinadas segundo a norma americana ASTM D-1037 - 1993. Para qualificação das chapas de flocos, os valores médios observados foram comparados com os valores mínimos exigidos pela norma comercial ANSI/A 208.1-1993, pelas normas CSA 0437-1993 e, segundo Santana e Pastore Júnior (1981), pela norma DIN 68 761 (1) - 1961. Contudo, o condicionamento das chapas foi em ambiente com temperatura média igual a $25^{\circ} \mathrm{C}$ e umidade relativa de $65 \%$. Posteriormente, as amostras destinadas à avaliação da estabilidade dimensional foram acondicionadas em ambiente cuja temperatura média foi igual a $25^{\circ} \mathrm{C}$ e umidade relativa igual de $95 \%$.

O experimento foi analisado admitindo-se um delineamento inteiramente casualizado, com 10 tratamentos e duas repetições. Os resultados dos testes das variáveis foram submetidos à análise de variância (ANOVA).

Quadro 1 - Tratamentos

Table 1 - Treatments

\begin{tabular}{cccc}
\hline Tratamento $\begin{array}{c}\text { Tipo } \\
\text { de adesivo }\end{array}$ & $\begin{array}{c}\text { Teor } \\
\text { de adesivo } \\
(\%)\end{array}$ & $\begin{array}{c}\text { Espécie de } \\
\text { madeira/flocos }\end{array}$ \\
\hline 1 & Tanino E. grandis & 8 & Pinus \\
2 & Tanino E.grandis & 10 & Pinus \\
3 & Tanino E.grandis & 8 & E. grandis \\
4 & Tanino E.grandis & 10 & E. grandis \\
5 & Tanino E.pellita & 8 & Pinus \\
6 & Tanino E. pellita & 10 & Pinus \\
7 & Tanino E.pellita & 8 & E. grandis \\
8 & Tanino E. pellita & 10 & E. grandis \\
9 & "Uréia" & 8 & Pinus \\
10 & "Uréia" & 8 & E. grandis \\
\hline
\end{tabular}

R. Árvore, Viçosa-MG, v.28, n.4, p.571-582, 2004 


\section{RESULTADOS E DISCUSSÕES}

\subsection{Propriedades dos taninos e dos adesivos}

Os valores qualitativos e quantitativos dos taninos nas cascas de Eucalyptus grandis e Eucalyptus pellita são apresentados nos Quadros 2 e 3, respectivamente.

Nota-se no Quadro 2 que, com exceção do tratamento com $1,5 \%$ de sulfito de sódio, o aumento na temperatura de extração dos taninos elevou o rendimento dos sólidos totais. No entanto, em ambas as temperaturas a inclusão de sulfito de sódio na solução extratora ocasionou decréscimo no índice de Stiasny em relação ao tratamento com água pura.

Entre os tratamentos em que ocorreu a adição de sulfito de sódio, observou-se que tanto nas extrações a $70^{\circ} \mathrm{C}$ quanto a $100{ }^{\circ} \mathrm{C}$ houve acréscimo no índice de Stiasny à medida que aumentou a porcentagem de sulfito de sódio. Isso é interessante para a produção dos adesivos, pois, quanto maior o índice de Stiasny, menor a porcentagem de substâncias não-tânicas presentes nos extratos. A presença dessas substâncias proporciona problemas de viscosidade e resistência da linha de cola (PIZZI, 1994).

Analisando o efeito da temperatura, observouse que nas extrações a $70^{\circ} \mathrm{C}$ a adição do sulfito de sódio ocasionou aumento nas quantidades de taninos extraídos em torno de 9,$74 ; 21,41$; e $30,75 \%$, respectivamente, em 1,5;3,0; e 4,5\% de sulfito de sódio, em relação à extração apenas com água. Por esse motivo, para obter taninos da casca de Eucalypus grandis em quantidade suficiente para a fabricação dos adesivos, empregou-se uma solução contendo $4,5 \%$ de sulfito de sódio, na temperatura de $70^{\circ} \mathrm{C}$.

Na casca de Eucalyptus pellita, observou-se (Quadro 3) que, quando aumentaram a temperatura e a porcentagem de sulfito de sódio, aumentou também o rendimento em sólidos totais. O índice de Stiasny sofreu variações similares àquelas observadas na casca de Eucalyptus grandis, com maiores índices nos tratamentos realizados a $70^{\circ} \mathrm{C}$. Observou-se, ainda, um acréscimo em sólidos totais à medida que aumentou a porcentagem de sulfito de sódio. A extração apenas com água proporcionou os melhores índices de Stiasny em ambas as temperaturas de extração, porém não foi utilizada no preparo dos adesivos devido ao seu baixo rendimento em taninos. Para obtenção dos taninos das cascas de E. pellita em quantidade suficiente para a produção de adesivo, foram empregados à solução aquosa $4,5 \%$ de sulfito de sódio, e as extrações ocorreram à $100^{\circ} \mathrm{C}$.

As propriedades dos adesivos apresentadas no Quadro 4 evidenciam que o teor de sólidos dos adesivos tânicos de Eucalyptus pellita foi menor que o dos de Eucalyptus grandis, devido a uma maior proporção de água adicionada nesses taninos para a reação de sulfitação. Isso foi necessário porque, empregando a mesma proporção de água utilizada na sulfitação dos taninos extraídos da casca de Eucalyptus grandis, obtiveram-se adesivos muito viscosos, impossibilitando a sua aplicação na pistola pneumática.

Quadro 2 - Rendimento em taninos e substâncias não-tânicas da extração aquosa da casca de Eucalyptus grandis Table 2 - Tannic and non tannic aqueous solution yield for Eucalyptus grandis bark

\begin{tabular}{lrcccc}
\hline Tratamentos & Temp. ${ }^{\circ} \mathrm{C}$ & $\begin{array}{c}\text { Rend. em } \\
\text { sólidos (\%) }\end{array}$ & $\begin{array}{c}\text { Rend. em } \\
\text { taninos (\%) }\end{array}$ & $\begin{array}{c}\text { Rend. em } \\
\text { substâncias } \\
\text { não-tânicas (\%) }\end{array}$ & $\begin{array}{c}\text { Índice } \\
\text { Stiasny } \\
(\%)\end{array}$ \\
\hline Água pura & 70 & 17,60 & 12,88 & 4,72 & 73,20 \\
Água pura & 100 & 21,55 & 15,59 & 5,96 & 72,35 \\
Água $+1,5 \%$ sulfito & 70 & 22,65 & 14,27 & 8,38 & 63,01 \\
Água + 1,5\% sulfito & 100 & 22,23 & 13,81 & 8,42 & 62,15 \\
Água + 3\% sulfito & 70 & 25,20 & 16,39 & 8,81 & 65,07 \\
Água + 3\% sulfito & 100 & 26,74 & 17,29 & 9,45 & 64,67 \\
Água $+4,5 \%$ sulfito & 70 & 26,09 & 18,60 & 7,49 & 71,25 \\
Água $+4,5 \%$ sulfito & 100 & 26,84 & 18,47 & 8,37 & 68,84 \\
\hline
\end{tabular}


Nota-se, pelo Quadro 4, que as propriedades dos taninos das duas espécies estudadas foram alteradas pela sulfitação ácida, principalmente em relação à viscosidade, que foi bastante reduzida. Isso indica que, provavelmente, a sulfitação ácida dos taninos promoveu a hidrólise de carboidratos, gomas hidrocoloidais de peso molecular relativamente alto e também o rompimento das ligações interflavonóides $\left(\mathrm{C}_{4}-\right.$ $\mathrm{C}_{6}$ ou $\mathrm{C}_{4}-\mathrm{C}_{8}$ ) das unidades poliméricas dos taninos. A presença dessas gomas de alto peso molecular é responsável pela alta viscosidade característica do extrato tânico (PIZZI, 1994). Assim, Mori (2000) encontrou valores de viscosidade em Eucalyptus grandis acima de $6.000 \mathrm{cP}$, mesmo após a sulfitação ácida dos taninos. Contudo, analisando o Quadro 3, nota-se que os valores de viscosidade das duas espécies estudadas ficaram bem abaixo dos encontrados por esse autor, sendo a viscosidade dessas espécies igual a $427 \mathrm{cP}$ e $520 \mathrm{cP}$, respectivamente, emEucalyptus grandis e Eucalyptus pellita. As Figuras 2 e 3 ilustram a estrutura dos taninos condensados após a reação de sulfita- ção e os prováveis produtos da hidrólise ácida, respectivamente.

O tempo de gelatinização dos adesivos modificados foi maior que o dos adesivos à base de taninos não-modificados. Isso evidencia que a alta reatividade dos taninos com o formaldeído foi diminuída com a reação química.

No Quadro 5, mostram-se os parâmetros cinéticos dos adesivos à base de taninos sulfitados das duas espécies estudadas e do adesivo à base de uréiaformaldeído.

Pelo Quadro 5, pode-se verificar que os adesivos à base de taninos de Eucalyptus pellita tiveram os menores valores de entalpia em relação aos adesivos à base de taninos de Eucalyptus grandis. Essa diferença provavelmente foi ocasionada pelo menor teor de sólidos dos adesivos à base de taninos de $E u$ calyptus pellita. Essa diferença pode, ainda, ter sido ocasionada por uma provável diferença na constituição química do anel A da unidade flavonóide, que pode

Quadro 3 - Rendimento em taninos e substâncias não-tânicas da extração aquosa da casca de Eucalyptus pellita. Table 3 - Tannic and non-tannic aqueous solution yield for Eucalyptus pellita bark

\begin{tabular}{lrcccc}
\hline Tratamentos & Temp. $^{\circ} \mathrm{C}$ & $\begin{array}{c}\text { Rend. em } \\
\text { sólidos (\%) }\end{array}$ & $\begin{array}{c}\text { Rend. em } \\
\text { taninos (\%) }\end{array}$ & $\begin{array}{c}\text { Rend. em } \\
\text { substâncias } \\
\text { não-tânicas (\%) }\end{array}$ & $\begin{array}{c}\text { Índice } \\
\text { Stiasny } \\
(\%)\end{array}$ \\
\hline Água pura & 70 & 6,31 & 4,58 & 1,75 & 72,54 \\
Água pura & 100 & 8,86 & 6,46 & 2,40 & 73,01 \\
Água +1,5\% sulfito & 70 & 9,42 & 6,54 & 2,88 & 69,40 \\
Água + 1,5\% sulfito & 100 & 10,60 & 7,07 & 3,53 & 66,73 \\
Água + 3\% sulfito & 70 & 9,73 & 7,07 & 2,66 & 72,69 \\
Água + 3\% sulfito & 100 & 13,09 & 7,88 & 4,21 & 67,84 \\
Água + 4,5\% sulfito & 70 & 10,96 & 11,38 & 2,97 & 72,99 \\
Água $+4,5 \%$ sulfito & 100 & 15,76 & & 4,38 & 72,19 \\
\hline
\end{tabular}

Quadro 4 - Propriedades dos adesivos utilizados na produção das chapas Table 4-Board adhesive properties

\begin{tabular}{lcccr}
\hline Tipo de adesivo & $\mathrm{pH}$ & Teor de sólidos (\%) & Tempo de gelatinização (s) & Viscosidade (cP) \\
\hline Uréia/formaldeído & 8 & 61,35 & 55 & 250 \\
Taninos não sulfitados $^{1}$ & 5,20 & 51,00 & 21 & $>6000$ \\
Taninos não sulfitados $^{2}$ & 6,63 & 49,70 & 19 & $>6000$ \\
Adesivo 1 & 4,94 & 43,61 & 53 & 427 \\
Adesivo 2 & 5,65 & 39,80 & 64 & 520 \\
\hline
\end{tabular}

Em que: ${ }^{1}=$ taninos de Eucalyptus grandis e ${ }^{2}=$ taninos de Eucalyptus pellita.

Adesivo 1 - adesivo de taninos sulfitados de Eucalyptus grandis. Adesivo 2 - adesivo de taninos sulfitados de Eucalyptus pellita 
ser floroglucinólica ou resorcinólica. A unidade floroglucinólica reage mais rapidamente com o formaldeído. Deve-se considerar ainda que, durante o aquecimento de 0 a $250^{\circ} \mathrm{C}$, os adesivos passam por uma série de modificações que incluem evaporação de água livre e formaldeído residual, evaporação de água de reação e, também, a reação entre oligômeros com a formação de ligações metilênicas, culminando com a cura, que é o ponto onde se forma o retículo polimérico rígido. Desse ponto de vista, provavelmente os adesivos de taninos de Eucalyptus pellita formaram menor número de ligações metilênicas devido à menor quantidade de sólidos para reagir, ocasionando menor extensão de cura e, como conseqüência, menor entalpia.
O adesivo comercial à base de uréia forneceu a menor temperatura de pico $(\mathrm{T})\left(136,44^{\circ} \mathrm{C}\right)$ e também uma baixa energia de ativação $(76,91 \mathrm{kj} / \mathrm{mol})$, o que implica menor gasto de energia. Os adesivos à base de taninos apresentaram altas temperaturas de pico e bandas largas de polimerização, começando a partir de $100{ }^{\circ} \mathrm{C}$ e terminando em torno de $200{ }^{\circ} \mathrm{C}$, não diferenciando muito dos valores encontrados por Mori (2000).

Segundo Bacelar (2001), a ordem de reação (N) é uma classificação pelo número de moléculas que atuam sobre a velocidade da reação. De forma geral, a ordem de reação $(\mathrm{N})$ aumentou com a elevação dos valores de $\mathrm{pH}$ dos adesivos, tendo o adesivo à base de uréia com maior valor de $\mathrm{pH}=8$ e com maior valor de $\mathrm{N}(2,7)$.<smiles>CC(C)(C)c1cc2c(cc1O)OC(c1ccc(O)c(O)c1)C(O)[C@@H]2C(C)(C)C</smiles><smiles>CC(C)(C)c1cc(C(C(O)C(c2ccc(O)c(O)c2)C(C)(C)C)C([S+](=O)[O-])C(C)(C)C)c(O)cc1O</smiles>

(Fonte/Source: MORI, 2000).

Figura 2 - Sulfitação dos taninos condensados.

Figure 2 - Suphytation of condensed tannins.

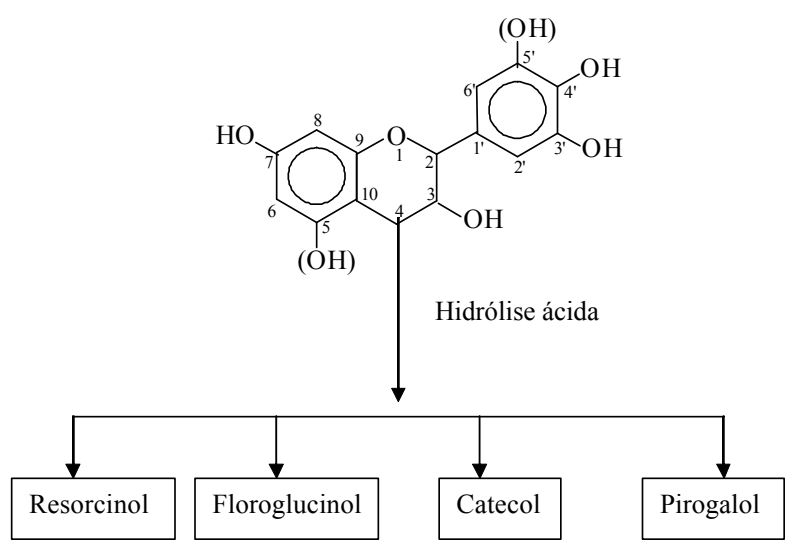

Figura 3 - Estrutura química dos monoflavonóides e respectivos produtos após a hidrólise.

Figure 3 -Chemical structure of monoflavonol and hydrolysis products.
Quadro 5 - Parâmetros cinéticos dos adesivos Table 5 -Adhesive kinetic parameters

\begin{tabular}{|c|c|c|c|c|}
\hline Adesivos & $\begin{array}{c}\Delta \mathrm{H} \\
(\mathrm{J} / \mathrm{g})\end{array}$ & $\begin{array}{c}\text { Temp. } \\
\left({ }^{\circ} \mathrm{C}\right)\end{array}$ & $\begin{array}{c}\mathrm{Ea} \\
(\mathrm{KJ} / \mathrm{mol})\end{array}$ & $\mathrm{N}$ \\
\hline \multicolumn{5}{|l|}{ Uréia } \\
\hline formaldeído & 172,46 & 136,44 & 76,91 & 2,7 \\
\hline \multicolumn{5}{|l|}{ Tanino } \\
\hline \multicolumn{5}{|l|}{ Eucalyptus } \\
\hline grandis (sul & o) 169,85 & 199,91 & 76,14 & 0,5 \\
\hline \multicolumn{5}{|l|}{ Tanino } \\
\hline \multicolumn{5}{|l|}{ Eucalyptus } \\
\hline pellita (sulf & 147,75 & 191,71 & 69,70 & 1,0 \\
\hline
\end{tabular}

Em que: Ea = energia de ativação, $\Delta \mathrm{H}=$ entalpia, $\mathrm{T}=$ temperatura de pico e N: ordem de reação. 


\subsection{Propriedades das chapas}

Os valores médios das densidades das chapas são listados no Quadro 6. As chapas foram produzidas tendo como meta densidade igual a $0,56 \mathrm{~g} / \mathrm{cm}^{3}$ para chapas de Pinus e $0,70 \mathrm{~g} / \mathrm{cm}^{3}$ para chapas de Eucalyptus grandis. A densidade média das chapas fabricadas com essa madeira foi inferior à desejada, provavelmente devido à aplicação de uma taxa de compressão inferior àquela necessária. Contudo, na mesma espécie, não se observou diferença estatística entre a densidade das chapas indicando que elas foram fabricadas de forma homogênea.

No Quadro 7, mostram-se os valores médios do teor de umidade de equilíbrio das chapas após o acondicionamento em temperatura ambiente.
Observa-se, pelo Quadro 7, que os teores de umidade de equilíbrio das chapas produzidas com adesivos de taninos modificados e madeira de Pinus foram estatisticamente iguais aos das chapas produzidas com o adesivo comercial de uréia (T9). Não houve diferenças significativas quanto à fonte de tanino e à porcentagem de adesivo utilizada nesses tratamentos. Contudo, nas chapas produzidas com madeira deEucalyptus grandis, as umidades de equilíbrio higroscópico dos tratamentos contendo adesivos de taninos modificados deEucalyptus pellita e Eucalyptus grandis não diferiram entre si, mas foram significativamente maiores do que a dos tratamentos com o adesivo à base de uréia, pelo teste de Tukey a $5 \%$ de probabilidade.

Os valores médios da resistência mecânica das

Quadro 6 - Valores médios de densidade de chapas de Pinus elliottii e Eucalyptus grandis Table 6 - Pinus elliottii and Eucalyptus grandis mean board density

\begin{tabular}{|c|c|c|c|c|}
\hline Tratamento & Tipo de adesivo & $\begin{array}{c}\text { Teor de adesivo } \\
(\%)\end{array}$ & $\begin{array}{c}\text { Espécie de } \\
\text { madeira/flocos }\left(\mathrm{g} / \mathrm{cm}^{3}\right)\end{array}$ & Densidade* \\
\hline 1 & Tanino E. grandis & 8 & Pinus & 0,57 \\
\hline 2 & Tanino E. grandis & 10 & Pinus & 0,58 \\
\hline 3 & Tanino E. grandis & 8 & E. grandis & 0,67 \\
\hline 4 & Tanino E. grandis & 10 & E. grandis & 0,70 \\
\hline 5 & Tanino E.pellita & 8 & Pinus & 0,57 \\
\hline 6 & Tanino E. pellita & 10 & Pinus & 0,56 \\
\hline 7 & Tanino E. pellita & 8 & E. grandis & 0,69 \\
\hline 8 & Tanino E. pellita & 10 & E. grandis & 0,67 \\
\hline 9 & "Uréia" & 8 & Pinus & 0,56 \\
\hline 10 & "Uréia" & 8 & E. grandis & 0,68 \\
\hline
\end{tabular}

*Na mesma espécie de madeira, não se observou diferença significativa, pelo teste $\mathrm{F}$ a $5 \%$ de probabilidade.

Quadro 7 - Comparações entre os valores médios de umidade de equilíbrio higroscópico em chapas produzidas com madeira de Pinus elliottii e Eucalyptus grandis

Table 7 - Comparison between mean values of equilibrium moisture content of Pinus elliottii and Eucalyptus grandis boards

\begin{tabular}{|c|c|c|c|c|c|c|c|}
\hline \multicolumn{4}{|c|}{ Pinus elliottii } & \multicolumn{4}{|c|}{ Eucalyptus grandis } \\
\hline Tratamentos & $\begin{array}{c}\text { Teor de } \\
\text { adesivo }(\%)\end{array}$ & $\begin{array}{c}\text { Fonte de } \\
\text { tanino/adesivo }\end{array}$ & $\begin{array}{l}\text { Umidade } \\
(\%)\end{array}$ & Tratamentos & $\begin{array}{c}\text { Teor de } \\
\text { adesivo }(\%)\end{array}$ & $\begin{array}{c}\text { Fonte de } \\
\text { tanino/adesivo }\end{array}$ & $\begin{array}{l}\text { Umidade } \\
(\%)\end{array}$ \\
\hline 6 & 10 & E. pellita & $11,75 \mathrm{~A}$ & 7 & 8 & E. pellita & 12,00 \\
\hline 2 & 10 & E. grandis & $11,40 \mathrm{~A}$ & 8 & 10 & E. pellita & 12,00 \\
\hline 5 & 8 & E. pellita & $11,35 \mathrm{~A}$ & 4 & 10 & E. grandis & 11,95 \\
\hline 1 & 8 & E. grandis & $10,15 \mathrm{~A}$ & 3 & 8 & E. grandis & 11,50 \\
\hline 9 & 8 & "Uréia" & $10,00 \quad \mathrm{~A}$ & 10 & 8 & "Uréia" & $9,35 \mathrm{~B}$ \\
\hline C.V $=$ & & & 1,3 & C. $V=$ & & & 4,3 \\
\hline
\end{tabular}

As médias seguidas de uma mesma letra não diferem entre si, pelo teste de Tukey a 5\% de probabilidade. 
chapas são apresentados nos Quadros 8, 9 e 10. Todas as chapas fabricadas com flocos de Pinus apresentaram resistência à tração superior ao mínimo exigido pela norma ANSI/A 208-1-93. Somente as chapas fabricadas com flocos de $E$. grandis e adesivo de taninos de Eucalyptus pellita (T7) não atingiram o mínimo exigido pela norma.

Conforme pode ser observado no Quadro 8, as resistências à tração perpendicular das chapas de $E$. grandis produzidas com o adesivo de taninos de Eucalyptus grandis ou Eucalyptus pellita foram estatisticamente iguais. Portanto, a fonte de taninos e a quantidade de adesivo ( 8 e 10\%) não afetaram essa propriedade. No entanto, as chapas fabricadas com adesivo de taninos foram significativamente menos resistentes do que àquelas com adesivo à base de uréiaformaldeído (T10).

Nas chapas produzidas com madeira de Pinus, os tratamentos com taninos de Eucalyptus grandis e Eucalyptus pellita diferiram entre si e entre a testemunha. As chapas fabricadas com adesivo de uréia foram mais resistentes do que aquelas com adesivo à base de tanino de Eucalyptus grandis. Notou-se que dentro de cada fonte de tanino não houve diferença entre os dois teores de adesivos utilizados.

Verificou-se que, apesar da menor densidade, os valores médios de resistência à tração perpendicular das chapas produzidas com madeira de Pinus foram, de modo geral, superiores aos das feitas com madeira de $E$. grandis. Isso provavelmente se deveu à melhor taxa de compactação e ao maior contato entre os flocos de Pinus.

No Quadro 9, pode-se observar que os valores médios de módulo de ruptura apresentados tanto pelas chapas fabricadas com madeira de Pinus quanto pelas com madeira de $E$. grandis foram superiores ao mínimo exigido pela norma ANSI/A-208-1/93. Nota-se, nesse quadro, que nas chapas produzidas com madeira de E. grandis os tratamentos com maiores valores de (MOR) foram das chapas confeccionadas com adesivos de taninos da casca de Eucalyptus grandis em sua formulação.

Observa-se ainda, no Quadro 9, que as chapas fabricadas com adesivo de taninos Eucalyptus grandis e madeira de Pinus foram estatisticamente diferentes daquelas feitas com adesivo de taninoEucalyptus pellita, sendo a fonte de tanino de Eucalyptus grandis estatisticamente melhor. O teor de adesivo das chapas fabricadas com tanino de Eucalyptus grandis e madeira de Pinus influenciou, de forma positiva, o módulo de ruptura. No Quadro 9, verificou-se também que, nas chapas produzidas com madeira de E. grandis, os tratamentos com $10 \%$ de adesivos foram estatisticamente iguais ao tratamento produzido com adesivo à base de uréia e estatisticamente diferentes em relação aos tratamentos com $8 \%$ de adesivo de taninos. Isso indica a influência direta do teor de adesivo sobre a resistência a ruptura.

Quadro 8 - Comparações entre os valores médios de resistência à tração perpendicular em chapas produzidas com madeira de Pinus elliottii e Eucalyptus grandis

Table 8 -Comparisons between mean values of internal bond strength of Pinus elliottii and Eucalyptus grandis boards

\begin{tabular}{|c|c|c|c|c|c|c|c|}
\hline \multicolumn{4}{|c|}{ Pinus elliottii } & \multicolumn{4}{|c|}{ Eucalyptus grandis } \\
\hline Tratamentos & $\begin{array}{c}\text { Teor de } \\
\text { adesivo (\%) }\end{array}$ & $\begin{array}{c}\text { Fonte de } \\
\text { tanino/adesivo }\end{array}$ & $\begin{array}{l}\text { Tração } \\
(\mathrm{MPa})\end{array}$ & Tratamentos & $\begin{array}{c}\text { Teor de } \\
\text { adesivo (\%) }\end{array}$ & $\begin{array}{c}\text { Fonte de } \\
\text { tanino/adesivo }\end{array}$ & $\begin{array}{l}\text { Tração } \\
(\mathrm{MPa}) \\
\end{array}$ \\
\hline 9 & 8 & "Uréia" & $0,90 \mathrm{~A}$ & 10 & 8 & "Uréia" & $1,05 \mathrm{~A}$ \\
\hline 1 & 8 & E. grandis & $0,69 \mathrm{~B}$ & 4 & 10 & E. grandis & $0,44 \mathrm{~B}$ \\
\hline 2 & 10 & E. grandis & $0,67 \mathrm{~B}$ & 3 & 8 & E. grandis & $0,40 \mathrm{~B}$ \\
\hline 6 & 10 & E. pellita & $0,33 \mathrm{C}$ & 8 & 10 & E. pellita & $0,36 \mathrm{~B}$ \\
\hline 5 & 8 & E. pellita & $0,32 \mathrm{C}$ & 7 & 8 & E. pellita & $0,27 \mathrm{~B}$ \\
\hline C. $V=$ & & & 1,3 & C. $\mathrm{V}=$ & & & 4,3 \\
\hline
\end{tabular}

As médias ao longo da mesma coluna seguidas de uma mesma letra não diferem entre si, pelo teste de Tukey a 5\% de probabilidade 
Quadro 9 - Comparações entre os valores médios de resistência à flexão estática em chapas produzidas com madeira de Pinus elliottii e Eucalyptus grandis

Table 9 - Comparisons between mean values of static bending strength of Pinus elliottii and Eucalyptus grandis boards

\begin{tabular}{|c|c|c|c|c|c|c|c|c|c|}
\hline \multicolumn{5}{|c|}{ Pinus elliottii } & \multicolumn{5}{|c|}{ Eucalyptus grandis } \\
\hline Tratam. & $\begin{array}{c}\text { Teor de } \\
\text { adesivo } \\
(\%)\end{array}$ & $\begin{array}{c}\text { Fonte de } \\
\text { tanino/ } \\
\text { adesivo }\end{array}$ & $\begin{array}{l}\mathrm{MOE} \\
(\mathrm{MPa})\end{array}$ & $\begin{array}{l}\text { MOR } \\
(\mathrm{MPa})\end{array}$ & Tratam. & $\begin{array}{c}\text { Teor de } \\
\text { adesivo } \\
(\%)\end{array}$ & $\begin{array}{c}\text { Fonte de } \\
\text { tanino/ } \\
\text { adesivo }\end{array}$ & $\begin{array}{l}\mathrm{MOE} \\
(\mathrm{MPa})\end{array}$ & $\begin{array}{l}\text { MOR } \\
(\mathrm{MPa})\end{array}$ \\
\hline 2 & 10 & E. grandis & $3691,5 \mathrm{~A}$ & $36,93 \mathrm{~A}$ & 4 & 10 & E. grandis & $3619,7 \mathrm{~A}$ & $26,78 \mathrm{AB}$ \\
\hline 1 & 8 & E. grandis & $3640,9 \mathrm{~A}$ & $30,87 \mathrm{~B}$ & 3 & 8 & E. grandis & $3263,2 \mathrm{~B}$ & $22,96 \mathrm{~B}$ \\
\hline 9 & 8 & "Uréia" & $3211,8 \quad \mathrm{~B}$ & $28,94 \mathrm{~B}$ & 8 & 10 & E. pellita & $3220,5 \mathrm{~B}$ & $25,49 \mathrm{AB}$ \\
\hline 5 & 8 & E. pellita & $3015,7 \quad \mathrm{~B}$ & $22,40 \mathrm{C}$ & 10 & 8 & "uréia" & $3178,8 \mathrm{~B}$ & $29,33 \mathrm{~A}$ \\
\hline 6 & 10 & E. pellita & $2674,3 \quad \mathrm{C}$ & $18,19 \mathrm{C}$ & 7 & 8 & E. pellita & $2533,0 \mathrm{C}$ & $15,93 \mathrm{C}$ \\
\hline C.V $(\%)$ & & & 1,90 & 5,41 & C.V (\%) & & & 2,55 & 5,59 \\
\hline
\end{tabular}

As médias ao longo da mesma coluna seguidas de uma mesma letra não diferem entre si, pelo teste de Tukey a 5\% de probabilidade.

Os módulos de elasticidade de todos os tratamentos atenderam ao mínimo exigido pela norma ANSI/A 208.1/93. Os módulos de elasticidade observados nas chapas fabricadas com adesivos à base de uréiaformaldeído foram, de modo geral, inferiores aos obtidos pelas chapas com adesivos à base de taninos de Eucalyptus grandis. Os tratamentos T1 (chapa com $8 \%$ de adesivo tânico) e T2 (chapa com 10\% de adesivo tânico) fabricados com flocos de Pinus e taninos de Eucalyptus grandis foram estatisticamente diferentes dos tratamentos com tanino de Eucalyptus pellita. Verificou-se que os tratamentos T3 e T8 foram estatisticamente idênticos ao 10 e estatisticamente diferentes do 4 (chapa fabricada com flocos deE. grandis e $10 \%$ de adesivo à base de tanino deEucalyptus grandis). Notou-se que, nas chapas fabricadas com flocos de E. grandis e fonte de taninos de Eucalyptus grandis, o teor de adesivo influenciou, de forma direta, o módulo de elasticidade, sendo o adesivo tânico $10 \%$ estatisticamente melhor.

Os valores médios de resistência ao arrancamento de parafuso são representados no Quadro 10. Verifica-se, nesse quadro, que a resistência das chapas em todos os tratamentos foi superior ao mínimo estipulado pela norma ANSI/A 208.1-93, que exige, em chapas de baixa densidade, um valor de resistência igual ou superior a $550 \mathrm{~N}$ e em chapas de média densidade, resistência superior a $900 \mathrm{~N}$.

Quadro 10 - Comparações entre os valores médios de resistência ao arrancamento de parafusos em chapas produzidas com madeira de Pinus elliottii e Eucalyptus grandis

Table 10 - Comparisons between mean values of screw withdrawal resistance of Pinus elliottii and Eucalyptus grandis boards

\begin{tabular}{|c|c|c|c|c|c|c|c|}
\hline \multicolumn{4}{|c|}{ Pinus elliottii } & \multicolumn{4}{|c|}{ Eucalyptus grandis } \\
\hline Traatamentos & $\begin{array}{c}\text { Teor de } \\
\text { adesivo }(\%)\end{array}$ & $\begin{array}{c}\text { Fonte de } \\
\text { tanino/adesivo }\end{array}$ & $\begin{array}{c}\text { Arranque } \\
(\mathrm{N})\end{array}$ & Tratamentos & $\begin{array}{c}\text { Teor de } \\
\text { adesivo (\%) }\end{array}$ & $\begin{array}{c}\text { Fonte de } \\
\text { tanino/adesivo }\end{array}$ & $\begin{array}{c}\text { Arranque } \\
(\mathrm{N})\end{array}$ \\
\hline 6 & 10 & E. pellita & $1597,40 \mathrm{~A}$ & 10 & 8 & "Uréia" & $2072,70 \mathrm{~A}$ \\
\hline 2 & 10 & E grandis & $1582,70 \mathrm{~A}$ & 4 & 10 & E. grandis & $1852,20 \mathrm{~A}$ \\
\hline 9 & 8 & "Uréia" & $1489,60 \mathrm{~A}$ & 8 & 10 & E. pellita & $1808,10 \mathrm{~A}$ \\
\hline 5 & 8 & E. pellita & $1210,30 \mathrm{~B}$ & 3 & 8 & E. grandis & $1296,05 \mathrm{~B}$ \\
\hline 1 & 8 & E. grandis & $1131,90 \mathrm{~B}$ & 7 & 8 & E. pellita & $1141,70 \mathrm{~B}$ \\
\hline C. $V=$ & & & 4,72 & C. $\mathrm{V}=$ & & & 4,36 \\
\hline
\end{tabular}

As médias ao longo da mesma coluna seguidas de uma mesma letra não diferem entre si, pelo teste de Tukey a 5\% de probabilidade. 
Analisando os dados contidos no Quadro 10, observou-se que nas chapas produzidas com Pinus e $8 \%$ de adesivo não houve efeito significativo da fonte de taninos. Contudo, essas chapas foram menos resistentes do que àquelas fabricadas com adesivo à base de uréia. Observou-se ainda que as chapas fabricadas com $8 \%$ de adesivo comercial de uréia tiveram a mesma resistência do que as com $10 \%$ de adesivo de taninos de Eucalyptus pellita ou Eucalyptus grandis. Comparando o efeito do teor de adesivo à base de taninos, notou-se que as chapas fabricadas com $10 \%$ de adesivo foram estatisticamente melhores. Isso indica que o teor de adesivo influenciou diretamente a resistência ao arrancamento de parafuso.

Nas chapas fabricadas com madeira deE. grandis, verificou-se que ocorreu o mesmo efeito entre elas, sendo, portanto, os tratamentos 4,8 e 10 estatisticamente idênticos, e os tratamentos 3 (chapas produzidas com $8 \%$ de adesivos e com taninos de Eucalyptus grandis) e 7 (chapas produzidas com tanino deEucalyptus pellita e com $8 \%$ de adesivo) também não diferiram entre si, não tendo, portanto, diferença entre elas quanto à fonte de taninos. Os tratamentos com $10 \%$ de adesivo foram estatisticamente melhores que os demais.

Os valores médios de inchamento em espessura, absorção de água e expansão linear em chapas produzidas com madeira de Pinus e E. grandis são apresentados nos Quadros 11 e 12, respectivamente. Todas as chapas tiveram inchamento em espessura e absorção de água superiores àqueles permitidos pelas normas CSA 0437-93 e DIN. Os elevados valores observados neste experimento se devem, pelo menos em parte, ao fato de não se ter utilizado parafina em emulsão no preparo das chapas.

De acordo com os resultados apresentados pela análise de variância, o inchamento em espessura e absorção foi, de forma geral, afetado pelo tipo de tanino utilizado nas formulações. Analisando exclusivamente os tratamentos quanto à fonte de taninos, pôde-se verificar que, mesmo ultrapassando os valores máximos permitidos para inchamento em espessura e absorção, após 2 e 24 horas de imersão em água as formulações que continham adesivos de taninos deEucalyptus grandis incharam menos do que aqueles produzidos com taninos de Eucalyptus pellita. É provável que a maior absorção de água pelos tratamentos com Eucalyptus pellita seja devida à modificação química dos taninos, que os deixaram mais higroscópicos.

Analisando o Quadro 11, observa-se que nas chapas fabricadas com Pinus não houve diferença no inchamento em espessura entre os adesivos à base de taninos, após equilíbrio em umidades iguais a 65 e $95 \%$.Contudo, essas chapas tiveram inchamento em espessura superior ao daquelas fabricadas com adesivo à base de uréia.

Quadro 11 - Comparações entre os valores médios de inchamento em espessura em chapas produzidas com madeira de Pinus elliottii e Eucalyptus grandis

Table 11 - Comparisons between mean values of thickness swelling of Pinus elliottii and Eucalyptus grandis boards

\begin{tabular}{|c|c|c|c|c|c|c|c|c|c|}
\hline \multirow[b]{2}{*}{ Tratamento } & \multirow[b]{2}{*}{$\begin{array}{c}\text { Teor } \\
\text { adesivo } \\
(\%)\end{array}$} & \multicolumn{3}{|c|}{ Pinus elliottii } & \multirow[b]{2}{*}{ Tratamento } & \multirow[b]{2}{*}{$\begin{array}{c}\text { Teor } \\
\text { adesivo } \\
(\%)\end{array}$} & \multicolumn{3}{|c|}{ Eucalyptus grandis } \\
\hline & & $\begin{array}{c}\text { Inch. }{ }^{1} \\
\text { U.R } \\
(\%)\end{array}$ & $\begin{array}{l}\text { Inch. } \\
2 \mathrm{~h} \\
(\%)\end{array}$ & $\begin{array}{c}\text { Inch. } \\
24 \mathrm{~h} \\
(\%)\end{array}$ & & & $\begin{array}{c}\text { Inch. }^{1} \\
\text { U.R. } \\
(\%)\end{array}$ & $\begin{array}{l}\text { Inch. } \\
2 \mathrm{~h} \\
(\%)\end{array}$ & $\begin{array}{l}\text { Inch. } \\
24 \mathrm{~h} \\
(\%)\end{array}$ \\
\hline $5 * *$ & 8 & $18,1 \mathrm{~A}$ & $36,1 \mathrm{~A}$ & $57,4 \mathrm{~A}$ & $7 * *$ & 8 & $23,4 \mathrm{~A}$ & $33,4 \mathrm{~A}$ & $66,0 \mathrm{~A}$ \\
\hline $2 *$ & 10 & $18,0 \mathrm{~A}$ & $23,2 \mathrm{~B}$ & $34,0 \mathrm{C}$ & $3 *$ & 8 & $19,6 \mathrm{~B}$ & $26,8 \mathrm{AB}$ & $48,6 \mathrm{~B}$ \\
\hline $1 *$ & 8 & $17,6 \mathrm{~A}$ & $23,7 \mathrm{~B}$ & $38,4 \mathrm{BC}$ & $4 *$ & 10 & $18,2 \mathrm{~B}$ & $21,5 \mathrm{~B}$ & $35,5 \mathrm{C}$ \\
\hline $6 * *$ & 10 & $16,3 \mathrm{~A}$ & $28,4 \mathrm{AB}$ & $44,9 \mathrm{~B}$ & $8 * *$ & 10 & $17,8 \mathrm{~B}$ & $22,1 \mathrm{~B}$ & $41,4 \mathrm{BC}$ \\
\hline 9 & 8 & $8,8 \mathrm{~B}$ & $12,9 \mathrm{C}$ & $17,0 \mathrm{D}$ & 10 & 8 & $10,7 \mathrm{C}$ & $13,6 \mathrm{C}$ & $20,2 \mathrm{D}$ \\
\hline C.V $(\%)$ & - & 6,6 & 7,7 & 5,8 & C.V $(\%)$ & - & 6,2 & 8,0 & 5,9 \\
\hline
\end{tabular}

Em que Inch. = inchamento em espessura e Inch. ${ }^{1}=$ inchamento U.R. $>95 \%$.

* Taninos Eucalyptus grandis e ** Taninos Eucalyptus pellita.

As médias ao longo de toda a coluna seguidas de uma mesma letra, não diferem entre si, pelo teste de Tukey a 5\% de probabilidade.

R. Árvore, Viçosa-MG, v.28, n.4, p.571-582, 2004 
Quadro 12 - Comparações entre os valores médios de absorção de água em chapas produzidas com madeira de Pinus elliottii e Eucalyptus grandis

Table 12 - Comparisons between mean values of water absorption Pinus elliottii and Eucalyptus grandis boards

\begin{tabular}{|c|c|c|c|c|c|c|c|c|c|}
\hline \multirow[b]{2}{*}{ Tratamento } & \multicolumn{3}{|c|}{ Pinus elliottii } & \multirow[b]{2}{*}{$\begin{array}{c}\text { Absorção } \\
24 \mathrm{~h} \\
(\%)\end{array}$} & \multirow[b]{2}{*}{ Tratamento } & \multirow[b]{2}{*}{$\begin{array}{c}\text { Teor de } \\
\text { adesivo } \\
(\%)\end{array}$} & \multicolumn{2}{|c|}{ Eucalyptus grandis } & \multirow[b]{2}{*}{$\begin{array}{c}\text { Absorção } \\
24 \mathrm{~h} \\
(\%)\end{array}$} \\
\hline & $\begin{array}{c}\text { Teor de } \\
\text { adesivo } \\
(\%)\end{array}$ & $\begin{array}{c}\text { Fonte } \\
\text { taninos/ } \\
\text { adesivo }\end{array}$ & $\begin{array}{c}\text { Absorção } \\
2 \mathrm{~h} \\
(\%)\end{array}$ & & & & $\begin{array}{c}\text { Fonte } \\
\text { taninos/ } \\
\text { adesivo }\end{array}$ & $\begin{array}{c}\text { Absorção } \\
2 \mathrm{~h} \\
(\%)\end{array}$ & \\
\hline 5 & 8 & E. pellita & $88,0 \mathrm{~A}$ & $108,9 \mathrm{~A}$ & 7 & 8 & E. pellita & $60,7 \mathrm{~A}$ & $89,9 \mathrm{~A}$ \\
\hline 6 & 10 & E. pellita & $79,9 \mathrm{~A}$ & $98,9 \mathrm{AB}$ & 8 & 10 & E. pellita & $43,6 \mathrm{~B}$ & $69,5 \mathrm{~B}$ \\
\hline 9 & 8 & "Uréia" & $70,0 \mathrm{AB}$ & $76,4 \mathrm{ABC}$ & 3 & 8 & E. grandis & $42,7 \mathrm{~B}$ & $65,9 \mathrm{BC}$ \\
\hline 1 & 8 & E. grandis & $68,7 \mathrm{AB}$ & $85,0 \mathrm{BC}$ & 4 & 10 & E. grandis & $39,5 \mathrm{~B}$ & $59,6 \mathrm{C}$ \\
\hline 2 & 10 & E. grandis & $54,9 \mathrm{~B}$ & $69,6 \mathrm{C}$ & 10 & 8 & "Uréia" & $30,9 \mathrm{C}$ & $44,2 \mathrm{D}$ \\
\hline C.V\% & - & -- & 7,5 & 6,9 & C.V\% & - & -- & 2,4 & 3,2 \\
\hline
\end{tabular}

As médias ao longo de toda a coluna seguidas de uma mesma letra não diferem entre si, pelo teste de Tukey a 5\% de probabilidade.

Após duas horas de imersão, o inchamento em espessura das chapas fabricadas com Pinus não foi afetado pelo teor de adesivos de taninos. No entanto, quando se analisa o efeito da fonte de taninos, observa-se que o adesivo contendo tanino deEucalyptus pellita ocasionou maior inchamento em espessura, tanto nas chapas fabricadas com madeira de $E$. grandis quanto naquelas com madeira dePinus. Todas as chapas fabricadas com adesivo comercial foram mais estáveis em relação às feitas com adesivos tânicos.

As propriedades das chapas fabricadas com madeira de E. grandis foram afetadas pelo teor de adesivo, em ambas as fontes de taninos, sendo aquelas produzidas com $10 \%$ de adesivo mais estáveis tanto após duas horas quanto após 24 horas de imersão em água. As chapas fabricadas com adesivos à base de uréia foram estatisticamente melhores do que as produzidas com adesivos à base de taninos, tanto no que se refere à absorção de água quanto à estabilidade dimensional. De modo geral, os tratamentos com $8 \%$ de adesivo de taninos de Eucalyptus pellita resultaram em maiores inchamentos, tanto nas duas quanto nas 24 horas de imersão.

Os valores médios de absorção de água são apresentados no Quadro 12, onde se observa que, após a imersão por duas horas, as chapas fabricadas com madeira de Pinus e tratamentos utilizando adesivos de taninos de Eucalyptus pellita em sua formulação foram estatisticamente iguais aos tratamentos com uréia (T9), bem como as chapas fabricadas com $8 \%$ de adesivo de tanino obtido de Eucalyptus grandis. No entan- to, a absorção de água, por duas horas, da chapa fabricada com $10 \%$ de adesivo tânico deEucalyptus grandis foi significativamente menor que aquelas feitas com adesivo de tanino de Eucalyptus pellita, contudo não diferiram da testemunha. As chapas confeccionadas com madeira de E. grandis e adesivos tânicos absorveram significativamente mais água do que aquelas fabricadas com adesivos de uréia. A maior absorção foi observada na chapa contendo $8 \%$ de adesivo de taninos de Eucalyptus pellita.

$\mathrm{Na}$ absorção em água por 24 horas, observou-se que nas chapas feitas com madeira de Pinus não houve diferença significativa entre os tratamentos 5 e 6 , indicando que não ocorerreu influência do teor de adesivo. Provavelmente o teor de adesivo igual a $8 \%$ é adequado. Nota-se, também, que os tratamentos 1 e 2 (ambos com taninos de Eucalyptus grandis) não diferiram entre si. Contudo, as chapas fabricadas com adesivo tânico oriundo da casca de Eucalyptus pellita absorveram significativamente mais água do que as feitas com adesivos à base de taninos de Eucalyptus grandis. Nas chapas fabricadas com madeira de $E$. grandis, observou-se que não houve diferenças significativas na absorção de água após duas horas de imersão entre as chapas fabricadas com $10 \%$ de adesivo tânico de Eucalyptus pellita e aquelas confeccionadas com taninos de Eucalyptus grandis. No entanto, as chapas fabricadas com $8 \%$ de adesivo de taninos das cascas de Eucalyptus pellita diferiram significativamente das com $10 \%$ de adesivo tânico de Eucalyptus pellita e dos demais tratamentos, tanto de duas quanto de 24 horas de absorção.

R. Árvore, Viçosa-MG, v.28, n.4, p.571-582, 2004 
Analisando exclusivamente os tratamentos com adesivos de taninos de Eucalyptus grandis, observou-se que na absorção de duas e 24 horas não ocorreram diferenças quanto ao teor de adesivo aplicado.

Os valores médios de expansão linear indicaram que todos os tratamentos atenderam às especificações exigidas pela norma ANSI/A.1.208.93, que permite uma expansão linear máxima de $0,35 \%$. Além disso, não se verificou diferença significativa entre os tratamentos.

\section{CONCLUSÕES}

Os resultados evidenciaram a potencialidade do uso dos taninos das cascas de E. grandis e E. pellita na produção de adesivos para uso em chapas de composição para aplicação em interiores, onde a resistência à umidade não é exigida. Com base nos resultados, pôde-se chegar às seguintes conclusões:

- Os melhores rendimentos em taninos, tanto de Eucalyptus pellita (10,67\%) quanto de Eucalyptus grandis $(17,20 \%)$, foram obtidos na extração com $4,5 \%$ de sulfito de sódio.

- Em Eucalyptus pellita, a temperatura de melhor eficiência foi a de $100{ }^{\circ} \mathrm{C}$, enquanto em Eucalyptus grandis, de $70^{\circ} \mathrm{C}$.

- A sulfitação ácida provavelmente reduziu o peso molecular dos taninos e, conseqüentemente, a viscosidade dos adesivos, ficando num nível compatível com o preparo dos adesivos para produção de chapas.

- Por meio dos parâmetros cinéticos, concluiuse que os adesivos de taninos apresentaram altas temperaturas de pico e com larga banda de polimerização. Os taninos de Eucalyptus pellita exibiram menor valor de entalpia.

- As propriedades das chapas de flocos, na maioria das vezes, foram superiores àquelas estabelecidas pela norma ANSI/A 208.1-93. As propriedades das chapas fabricadas com adesivos de taninos de Eucalyptus grandis foram superiores àquelas feitas com adesivos de taninos de Eucalyptus pellita.

\section{REFERÊNCIAS BIBLIOGRÁFICAS}

AMERICAN NATIONAL STANDARD. ANS. Mat-formed wood particleboard : specification ANSI/A 208.1.1993. Gaithersburg: National Particleboards Association, 1993. 9p.

R. Árvore, Viçosa-MG, v.28, n.4, p.571-582, 2004
AMERICAN SOCIETY FOR TESTING AND MATERIALS. ASTM - Annual Book of ASTM Standards. v.04.09, 1993.

CANADIAN STANDARTS ASSOCIATION - CSA. OSB an waferboard. Ontario : CSA 0437 $-93,1993.18 \mathrm{p}$.

BARCELAR, C.S. Efeito da variação do pH e da temperatura de síntese no desempenho de adesivo à base de creosoto vegetal desmetiladoformaldeído. 2001. 43f. Dissertação (Mestrado em Ciência Florestal) - Universidade Federal de Viçosa, Viçosa, 2001.

HASLAM, E. Chemistry of vegetable tannins. New York: Academic Press, 1966. 177p.

HERGERT, H.L. Condensed tannins in adhesives: introduction and historical perspectives. In: HEMINGWAY, R.W. et al. (Eds.) Adhesives from renewable resources. Washington, D.C.: American Chemical Society, 1989. p. 155-171. (ACS Symposium).

MORI,F.A. Caracterização parcial dos taninos da casca e dos adesivos de três espécies de $\boldsymbol{E}$. grandis. 2000. 73f. Tese (Doutorado em Ciência Florestal). Universidade Federal de Viçosa, Viçosa, 2000.

PIZZI, A.; MITTAL, K.L. Handbook of adhesive technology. New York: Marcell Dekker, 1994. 680p.

PIZZI, A. Advanced wood adhesives technology. New York: Marcell Dekker, 1994. 289 p.

PIZZI, A. Wood adhesives: chemistry and technology. New York: Marcell Dekker, 1983. 364p.

PORTER, L.J. Flavans and proanthocyanidins. In: HARBORNE, J.B. (Ed.) The flavonoids: advances in research since 1980. New York: Chapman and Hall, 1988. 621 p.

SANTANA, M. A. E.; PASTORE JÚNIOR, F. Adesivo de tanino-formaldeído para aglomerados. Brasília: Instituto Brasileiro de Desenvolvimento Florestal, 1981. (Série técnica, 2)

WATERMAN, P.G.; MOLE, S. analysis of phenoloc plant metabolites. London: Blackwell Scientific Publications, 1994. 